\title{
Effect of Dopant Structure on Birefringences and Symmetric Tensor Invariants of Lyotropic Nematic Phases
}

\author{
Erol Akpinar \\ Department of Chemistry, Faculty of Arts and Sciences, Bolu Abant Izzet Baysal University, 14030, Golkoy, Bolu, \\ Turkey \\ akpinar_e@ibu.edu.tr
}

Received: 9 March 2018

Accepted: 12 June 2018

DOI: $10.18466 /$ cbayarfbe.403800

\begin{abstract}
In this study, we investigated the effect of the dopant structure on the birefringences and the symmetric tensor invariants of lyotropic discotic nematic $\left(\mathrm{N}_{\mathrm{D}}\right)$, calamitic nematic $\left(\mathrm{N}_{\mathrm{C}}\right)$ and biaxial nematic $\left(\mathrm{N}_{\mathrm{B}}\right)$ phases. Lyotropic host mixture consisted of potassium laurate $(\mathrm{KL}) / \mathrm{decanol}(\mathrm{DeOH}) /$ water. Some dopant molecules were added to this host mixture to obtain quaternary mixtures and to investigate the nematic phase properties by polarizing optical microscopy and laser conoscopy. It was found that the dopant molecules with aromatic ring affect the birefringences and symmetric tensor invariants more efficiently than those with straight alkyl chain.
\end{abstract}

Keywords: Lyotropic liquid crystals, nematic phases, birefringences, symmetric tensor invariants, laser conoscopy, polarizing optical microscopy.

\section{Introduction}

Properties of lyotropic nematic phases (LNPs) have been extensively investigated for several years to understand their formation mechanism and to find which factors affect obtaining the different nematic phases [1-4]. Their main property, which attracts the attention of the researchers, is that the local directors of their building blocks, so-called "micelles", exhibit average preferred alignment in a certain direction along the whole sample. Because of this, there is a long-range orientational order in the LNPs. The direction of preferred alignment in the LNPs is called the "phase director" or "optical axis", $\hat{n}$ [5]. From this respect, there are two different director alignments, either parallel or perpendicular to the magnetic field direction. The former and latter ones are also known as planar and homeotropic alignments, respectively.

Three different LNPs are defined in the literature. They are discotic nematic $\left(\mathrm{N}_{\mathrm{D}}\right)$, calamitic nematic $\left(\mathrm{N}_{\mathrm{C}}\right)$ and biaxial nematic $\left(\mathrm{N}_{\mathrm{B}}\right)$ phases [6-9]. From the optics point of view, the first two ones are uniaxial and the latter one is biaxial. In the $\mathrm{N}_{\mathrm{D}}\left(\mathrm{N}_{\mathrm{C}}\right)$ phase, the director of the phase is in the perpendicular (parallel) direction to the magnetic field. In other words, the $\mathrm{N}_{\mathrm{D}}$ phases are characterized by their homeotropic textures under the polarizing optical microscope (POM), i.e. a dark texture is observed if the sample is well-aligned by the effect of an external magnetic field. However, in the case of the $\mathrm{N}_{\mathrm{C}}$ phase, the texture is bright and colorful, depending on the birefringence of the sample. Thus, the $\mathrm{N}_{D}$ and $\mathrm{N}_{C}$ phases are easily recognizable from one another by the POM.
Experimental studies indicated that the $\mathrm{N}_{\mathrm{B}}$ phase is an intermediate phase between other two uniaxial phases in the phase diagrams of lyotropic mixtures [8,9]. Its microscopic texture is bright like the $\mathrm{N}_{\mathrm{C}}$ phase with different birefringences. So, the $\mathrm{N}_{\mathrm{B}}$ phase cannot easily be differentiated from the $\mathrm{N}_{C}$ phase. A better way is to measure the birefringences of all nematic phases as a function of temperature. It is well-known from experimental studies [10] and from theoretical predictions $[11,12]$ that the uniaxial-to-biaxial phase transitions are of second order as described by the meanfield theory.

The LNPs are also characterized by a symmetric tensor order parameter $[11,13]$. They have the same symmetry of the optical dielectric tensor, $\overleftrightarrow{\epsilon}$, which can be chosen as the order parameter $[14,15]$. The anisotropic part of $\overleftrightarrow{\epsilon}$ is related to the birefringences and the symmetric tensor invariants of the tensor order parameters, $\sigma_{2}$ and $\sigma_{3}$ [15].

In our previous study, we examined the effect of polar parts of some dopants on the nematic phase properties of potassium laurate $(\mathrm{KL}) /$ decanol $(\mathrm{DeOH}) /$ water lyotropic mixture [16]. In the present study, we are reporting the effect of non-polar parts of dopant structure on the birefringences and symmetric tensor invariants of lyotropic mixtures presenting uniaxial and biaxial nematic phases. The host lyotropic nematic mixture was chosen from the literature [16] and some different dopants were added to this mixture. Polarizing optical microscopy and laser conoscopy techniques were used to characterize the properties of the nematic phases. The 
results indicate that dopant structure is an important parameter on birefringences and symmetric tensor invariants.

\section{Materials and Methods}

\subsection{Chemicals}

All chemicals (toluene, benzyl alcohol, benzoic acid, dodecane, dodecanol and dodecanoic acid) were commercially purchased from Sigma, Merck and Aldrich in high purities $(99 \%)$. The surfactant molecule, potassium dodecanoate or laurate $(\mathrm{KL})$, was synthesized according to the procedure given in the literature [17]. Ultrapure water was provided by Millipore Direct-Q3 UV $\left(18.2 \mathrm{M} \Omega . \mathrm{cm}\right.$ of resistivity at $\left.25^{\circ} \mathrm{C}\right)$ for the preparation of lyotropic liquid crystal samples.

\subsection{Sample Preparation}

Lyotropic liquid crystalline samples were prepared by weighing the constituents in appropriate amounts into the well-closed test tubes. They were homogenized well by applying vortex and centrifuging occasionally. To obtain well-aligned nematic phases, a small amount of waterbased ferrofluid was added into the lyotropic mixtures as $1 \mu \mathrm{L}$ ferrofluid/1 g mixture.

\subsection{Polarizing Optical Microscopy}

Textural analyses of lyotropic samples were performed under the polarizing optical microscope. First, lyotropic samples were transferred into the flat microscope capillaries of 0.2 or $0.3 \mathrm{~mm}$ of thickness. Then, the capillaries were placed in a precise temperature control unit (a Linkam T95-PE temperature controller with a temperature stability of, at least, $0.01^{\circ} \mathrm{C}$ and LTS120E heating/freezing stage) with water circulation (Polyscience SD07R with an accuracy of $\pm 0.04^{\circ} \mathrm{C}$ ). By this way, a homogeneous heat distribution in the temperature control unit was provided.

\subsection{Laser Conoscopy}

Laser conoscopy was used to determine the temperature dependence of birefringences of three nematic phases and uniaxial-to-biaxial phase transition temperatures. For these measurements, the samples were put between two $2.5 \mathrm{~cm}$ (in diameter) optical glasses (Helma) which were separated by $2.5 \mathrm{~mm}$ (in thickness) of O-ring (Helma). Thus, $2.5 \mathrm{~mm}$ thick samples were obtained for the laser conoscopy measurements. The temperature was controlled by a Lakeshore 335 model temperature controller (with a Pt102 sensor and an accuracy of $\pm 0.001{ }^{\circ} \mathrm{C}$ ) and the heat distribution to all system was provided by a water circulating bath (Polyscience AD07R with an accuracy of $\pm 0.01{ }^{\circ} \mathrm{C}$ ). The laboratory frame axes were defined as follows: the horizontal plane was defined by the two orthogonal axes $\mathrm{x}$ and $\mathrm{y}$; the magnetic field $(\sim 2.3 \mathrm{kG})$ was aligned along the axis $\mathrm{x}$; axis $\mathrm{z}$ was vertical and parallel to the laser beam propagation direction.

\section{Results and Discussion}

\subsection{Polarizing Optical Microscopy}

The lyotropic mixture compositions are given in Table 1 . The same amount of dopant molecules as mole fraction were added to the lyotropic host nematic mixture $\mathrm{KL} /$ decanol (DeOH)/water. The dopant molecules can be divided into two different series, Figure 1. In the first series (toluene, benzyl alcohol and benzoic acid), the dopants include the aromatic rings. In the second series, (dodecane, dodecanol and dodecanoic acid), dopant molecules have straight aliphatic alkyl chains.

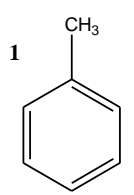

Toluene

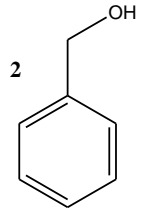

Benzyl alcohol<smiles>O=C(O)c1ccccc1</smiles>

Benzoic acid

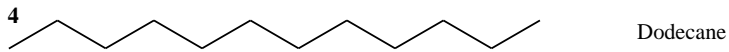

Dodecanol<smiles>CCCCCCCCCCCC(=O)O</smiles>

Figure 1. Molecular structures of dopant molecules studied in this study.

Table 1. Compositions of the lyotropic mixtures. $X$ corresponds to the mole fractions of the constituents of the mixtures in percent.

\begin{tabular}{|c|c|l|l|l|l|}
\hline Mixture & Dopant & $\mathbf{X}_{\text {KL }}$ & $\mathbf{X}_{\text {DeOH }}$ & $\mathbf{X}_{\text {water }}$ & $\mathbf{X}_{\text {dopant }}$ \\
\hline $\mathrm{m} 0$ & ----- & 3.316 & 1.212 & 95.472 & 0.000 \\
\hline $\mathrm{m} 1$ & $\mathbf{1}$ & 3.315 & 1.211 & 95.421 & 0.053 \\
\hline $\mathrm{m} 2$ & $\mathbf{2}$ & 3.315 & 1.211 & 95.421 & 0.053 \\
\hline $\mathrm{m} 3$ & $\mathbf{3}$ & 3.315 & 1.211 & 95.421 & 0.053 \\
\hline $\mathrm{m} 4$ & $\mathbf{4}$ & 3.315 & 1.211 & 95.421 & 0.053 \\
\hline $\mathrm{m} 5$ & $\mathbf{5}$ & 3.315 & 1.211 & 95.421 & 0.053 \\
\hline $\mathrm{m} 6$ & $\mathbf{6}$ & 3.315 & 1.211 & 95.421 & 0.053 \\
\hline
\end{tabular}

The nematic textures of the lyotropic mixtures were characterized by polarizing optical microscope. Figure 1 shows their characteristic non-aligned schlieren textures obtained from the lyotropic host mixture $\mathrm{KL} / \mathrm{DeOH} /$ water (mixture, $\mathrm{m} 0$ ). Other lyotropic mixtures exhibited similar textures. In the case of the presence of the magnetic field $(\sim 0.9 \mathrm{kG})$, homeotropic and planar textures were obtained for $\mathrm{N}_{\mathrm{D}}$ and $\mathrm{N}_{\mathrm{C}}$, respectively. 


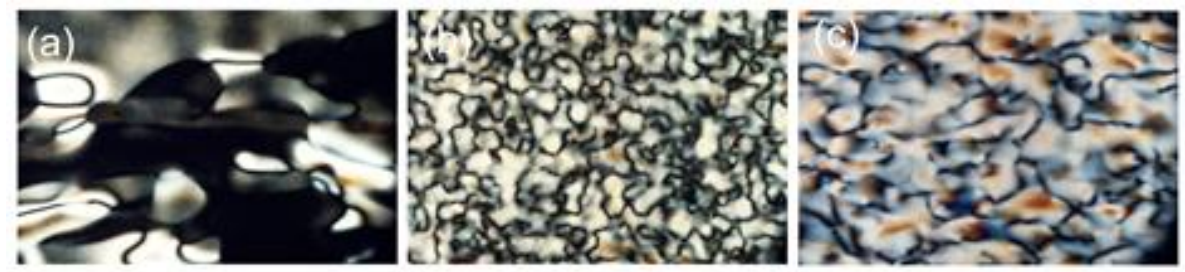

Figure 2. Characteristic schlieren textures of nematic phases for the mixture m0: (a) $N_{D}$ phase, (b) $N_{B}$ phase and (c) $\mathrm{N}_{\mathrm{C}}$ phase.

\subsection{Laser Conoscopy}

Laser conoscopy allows us to calculate the two main birefringences $\left(\Delta \mathrm{n}=\mathrm{n}_{\mathrm{y}}-\mathrm{n}_{\mathrm{x}}\right.$ and $\delta \mathrm{n}=\mathrm{n}_{\mathrm{z}}-\mathrm{n}_{\mathrm{y}}$, where $\mathrm{n}_{\mathrm{x}}, \mathrm{n}_{\mathrm{y}}$ and $\mathrm{n}_{\mathrm{z}}$ are the refractive indices along the axes $\mathrm{x}, \mathrm{y}$ and $\mathrm{z}$, respectively.) of the nematic phases as a function of temperature with high accuracy [10]. The key point in this technique is that the nematic samples have to be wellaligned by the effect of the external magnetic field. To do so, the addition of the ferrofluid to the lyotropic mixtures helps sample alignment in short time. The birefringences of the nematic phases were carried out starting from the $\mathrm{N}_{\mathrm{D}}$ phase. By changing the temperature, the uniaxial-tobiaxial phase transitions were determined. The characteristic laser conoscopy patterns were observed on the screen as shown in Figure 3 (For further information about the laser conoscopy, please see the Ref. [18]).
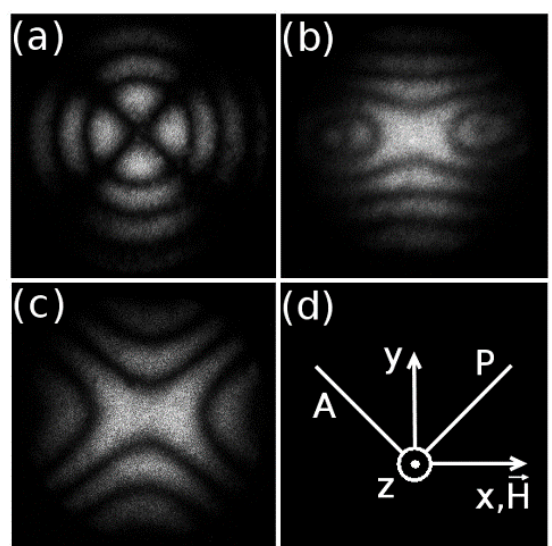

Figure 3. Laser conoscopy patterns of well-aligned (a) $\mathrm{N}_{\mathrm{D}}$, (b) $\mathrm{N}_{\mathrm{B}}$ and (c) $\mathrm{N}_{\mathrm{C}}$ phases. (d) shows the experimental setup, where $\mathrm{x}, \mathrm{y}$ and $\mathrm{z}$ are laboratory frame axes, $\mathrm{P}$ polarizer, A analyzer and $\mathrm{H}$ magnetic field direction.

The symmetric tensor invariants $\sigma_{1}, \sigma_{2}$ and $\sigma_{3}$ of the LNPs can be evaluated from laser conoscopy. These invariants are related to the diagonal elements of the anisotropic part of the dielectric tensor by the following equations $[10,15]$ :

$$
\begin{aligned}
& \sigma_{1}=\epsilon_{\mathrm{a} 1}+\epsilon_{\mathrm{a} 2}+\epsilon_{\mathrm{a} 3} \\
& \sigma_{2}=\frac{2}{3}\left(\epsilon_{a 1}^{2}+\epsilon_{a 2}^{2}+\epsilon_{a 3}^{2}\right) \\
& \sigma_{3}=4 \epsilon_{\mathrm{a} 1} \epsilon_{\mathrm{a} 2} \epsilon_{\mathrm{a} 3}
\end{aligned}
$$

where the first invariant $\sigma_{1}$ is zero. $\epsilon_{\mathrm{a} 1}, \epsilon_{\mathrm{a} 2}$ and $\epsilon_{\mathrm{a} 3}$ are calculated by using the birefringences values according to the equations $(3.4-3.6)[10,15]$ :

$$
\begin{aligned}
& \epsilon_{\mathrm{a} 1}=-\frac{4\langle\mathrm{n}\rangle}{3}\left(\Delta \mathrm{n}+\frac{\delta \mathrm{n}}{2}\right) \\
& \epsilon_{\mathrm{a} 2}=\frac{2\langle\mathrm{n}\rangle}{3}(\Delta \mathrm{n}-\delta \mathrm{n}) \\
& \epsilon_{\mathrm{a} 3}=\frac{4\langle\mathrm{n}\rangle}{3}\left(\frac{\Delta \mathrm{n}}{2}+\delta \mathrm{n}\right)
\end{aligned}
$$

where $\langle n\rangle$ is the average of the refraction index of the lyotropic mixture exhibiting the nematic phase and it can be measured by Abbe's refractometer.

Before discussing the role of the dopant molecules on the properties of the nematic phases, we determined the temperature dependence of the birefringences and symmetric tensor invariants and also the dependence of the $\sigma_{3}$ on the $\sigma_{2}$ for the ternary host mixture $\mathrm{KL} / \mathrm{DeOH} /$ water from the laser conoscopy, Figure 4. The similar calculations were carried out for the mixtures with the dopants and the results are given in Figures 5-8. Notice that the temperature dependences of birefringences of the ternary mixture and the quaternary mixture doped benzoic acid were reported for the first time in our previous study [16]. In the frame of this study, we repeated the same measurements for them and the results are in a good agreement with the previous ones. 

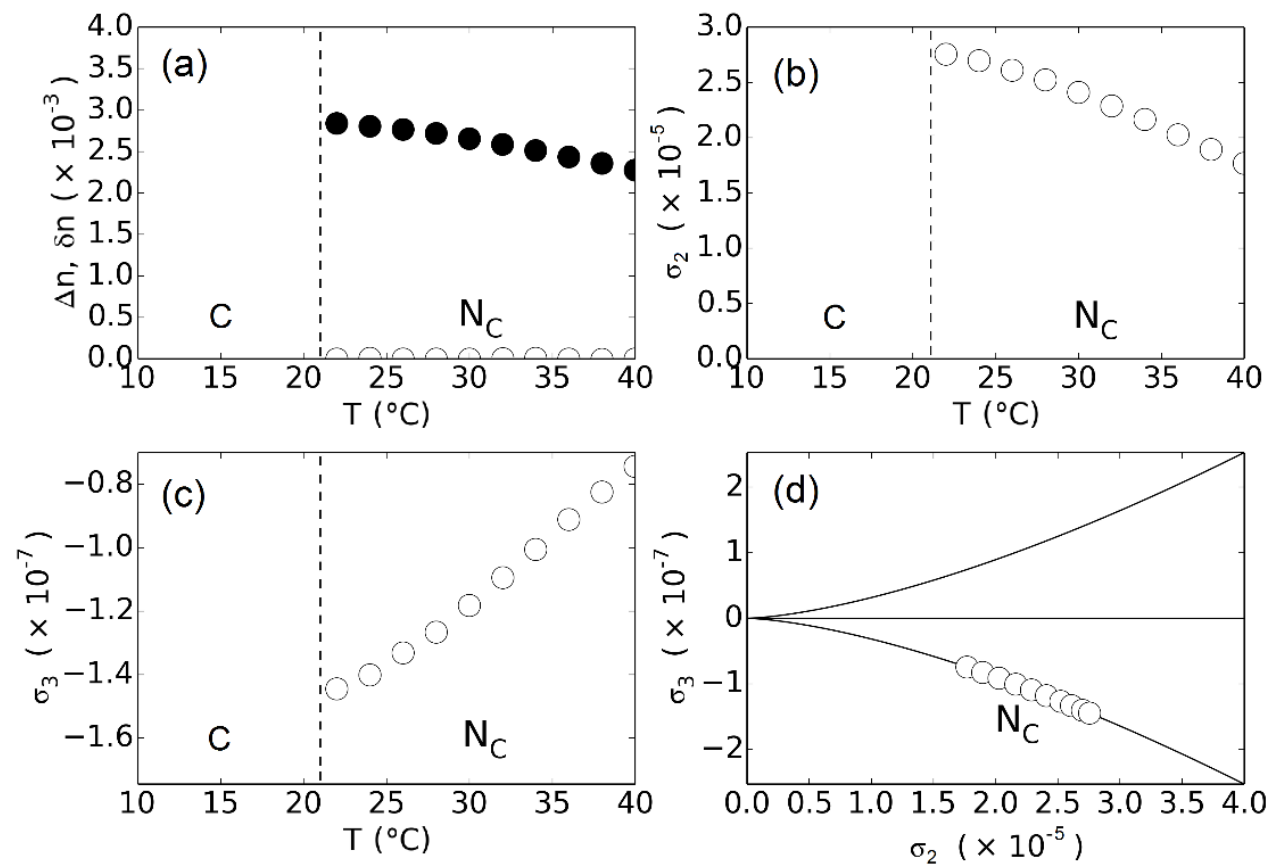

Figure 4. Laser conoscopy results of the ternary mixture $(\mathrm{m} 0)$ : temperature dependences of (a) the birefringences, (b) the $\sigma_{2}$ and (c) the $\sigma_{3}$. (d) The plot of $\sigma_{3}$ against $\sigma_{2}$. C represents non-nematic region.
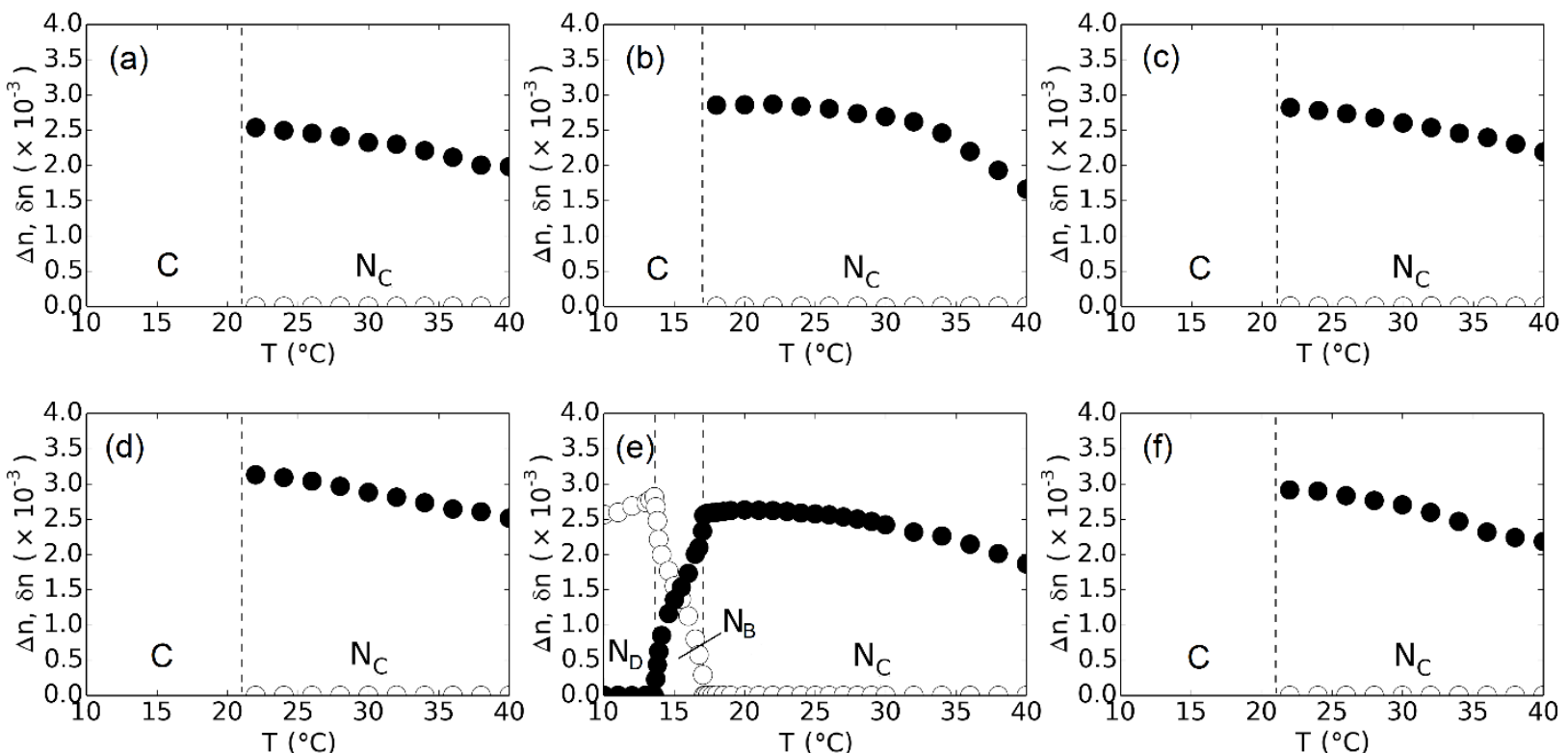

Figure 5. The temperature dependences of the birefringences for the KL/DeOH/water/dopant: (a) toluene, (b) dodecane, (c) benzyl alcohol, (d) dodecanol, (e) benzoic acid and (f) dodecanoic acid. 
Celal Bayar University Journal of Science
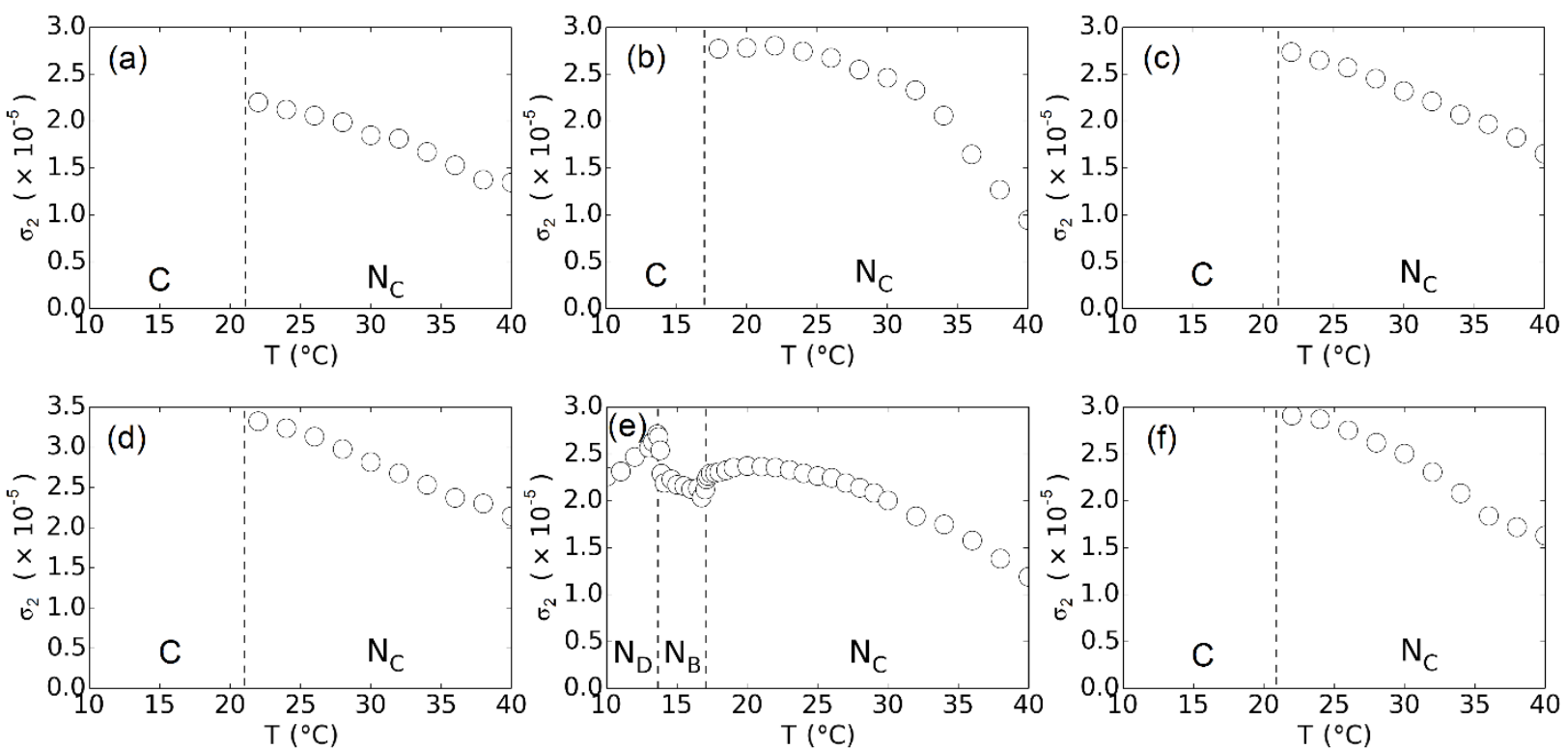

Figure 6. The temperature dependences of the symmetric tensor invariant $\sigma_{2}$ birefringences for the $\mathrm{KL} / \mathrm{DeOH} /$ water/dopant mixtures: (a) toluene, (b) dodecane, (c) benzyl alcohol, (d) dodecanol, (e) benzoic acid and (f) dodecanoic acid.
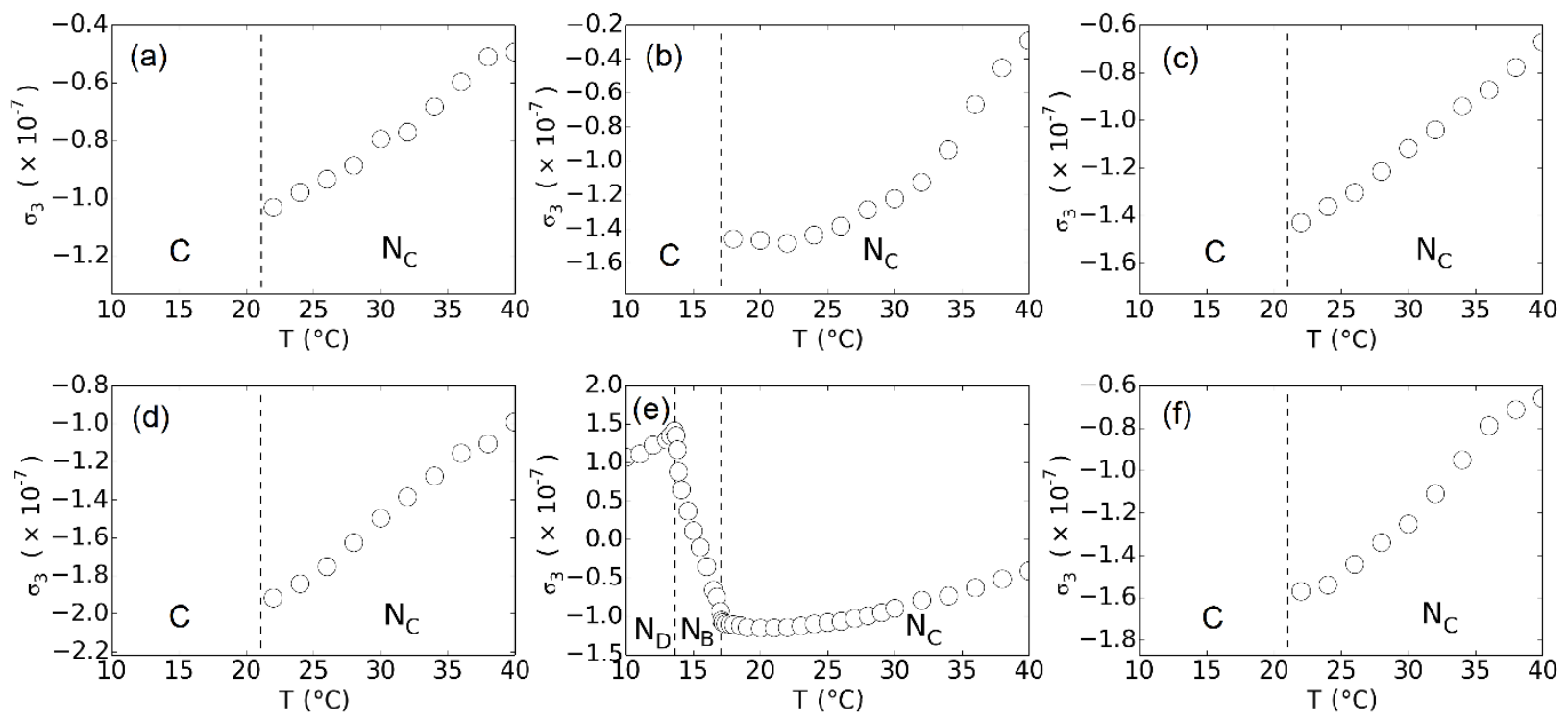

Figure 7. The temperature dependences of the symmetric tensor invariant $\sigma_{3}$ birefringences for the KL/DeOH/water/dopant mixtures: (a) toluene, (b) dodecane, (c) benzyl alcohol, (d) dodecanol, (e) benzoic acid and (f) dodecanoic acid. 

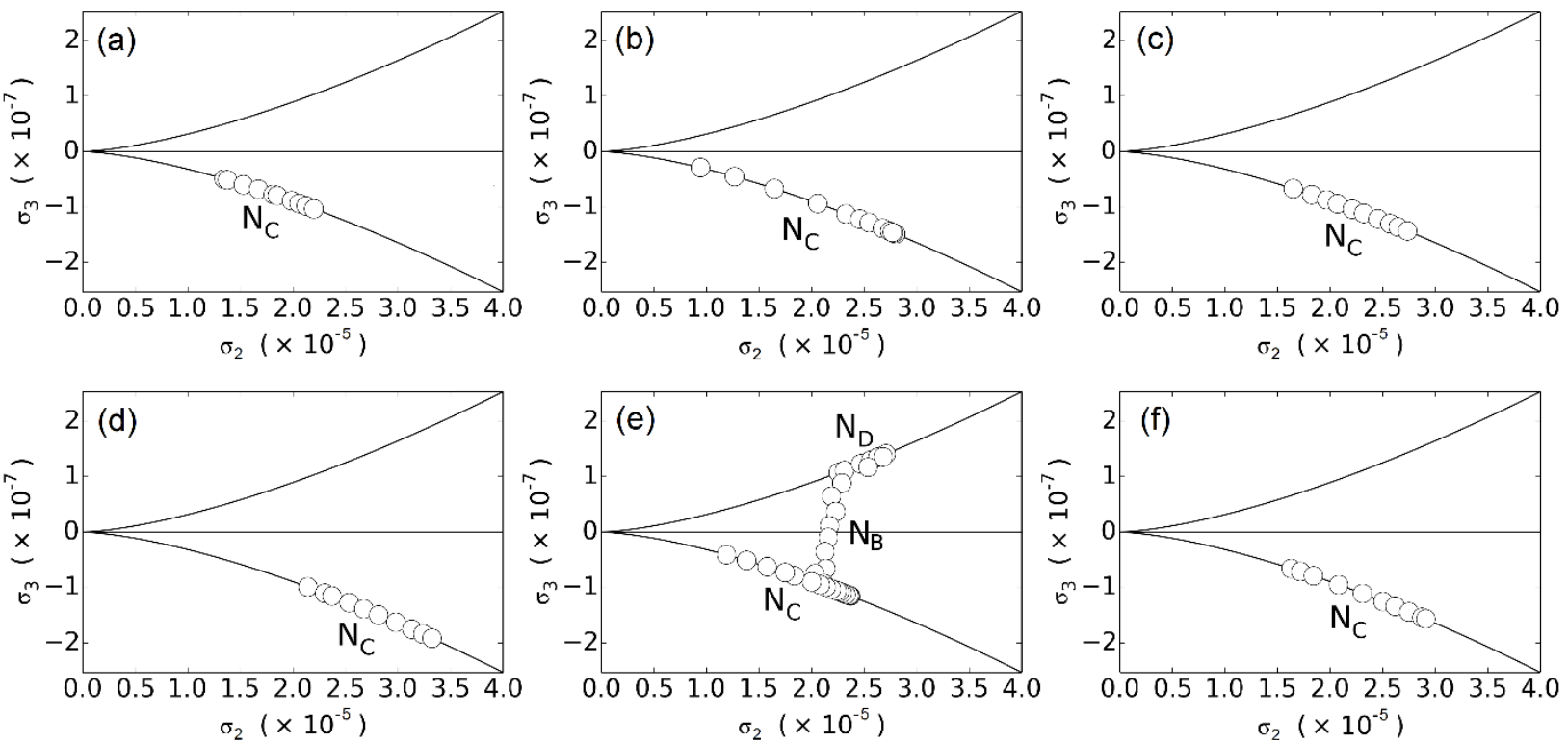

Figure 8. The variation of the $\sigma_{3}$ with the $\sigma_{2}$ for the KL/DeOH/water/dopant mixtures: (a) toluene, (b) dodecane, (c) benzyl alcohol, (d) dodecanol, (e) benzoic acid and (f) dodecanoic acid.

If the birefringences values of the ternary host mixture (Figure 4a) are compared with those of the quaternary mixtures (Figure 5), the dopant molecules with straight aliphatic chain (dodecane, dodecanol, dodecanoic acid) do not change, within the experimental error, not only the birefringences values but also the nematic phase type of the ternary mixture. However, the dopant molecules with aromatic ring (toluene, benzyl alcohol and benzoic acid) decrease the birefringences of the ternary mixture from $\sim 3.0 \times 10^{-3}$ to $\sim 2.5 \times 10^{-3}$. Because the smaller the birefringences means the smaller micelles as proved by small-angle x-ray scattering [19], the addition of the dopants with aromatic ring causes the decrease in the micelle size. Furthermore, as seen from the Figure 5e, the benzoic acid gives rise to the formation of $\mathrm{N}_{D}$ and $\mathrm{N}_{B}$ phases in addition to the $\mathrm{N}_{C}$ phase with respect to the ternary mixture. This result may be attributed to relatively strong interactions of the surfactant head groups with the carboxyl group of that molecule.

Similar to the birefringences results, while the symmetric tensor invariants $\sigma_{2}$ and $\sigma_{3}$ of the quaternary mixtures of dodecane, dodecanol and dodecanoic acid exhibit almost same temperature dependences, the quaternary mixtures of toluene, benzyl alcohol and benzoic acid show different temperature dependences than the ternary mixture.

\section{Conclusion}

In the present work, we studied the effect of the dopant structure on the birefringences and symmetric tensor invariants of the lyotropic nematic phases. The results indicated that the dopants including aromatic ring affect the birefringences and the invariants more efficiently than those with straight aliphatic alkyl chain. In addition, while the former ones change the micelle sizes, the latter ones not.

\section{Acknowledgements}

We would like to thank The Scientific and Technological Research Council of Turkey (TUBITAK, grant number: 114Z031) and Abant Izzet Baysal University Directorate of Research Projects Commission (BAP) [2016.03.03.988] for supporting this present study.

\section{References}

1. Akpinar, E, Reis, D, Neto, A.M.F, Effect of alkyl chain length of alcohols on nematic uniaxial-to-biaxial phase transitions in a potassium laurate/alcohol $/ \mathrm{K}_{2} \mathrm{SO}_{4} /$ water lyotropic mixture, Liquid Crystals, 2012, 39 (7), 881-888.

2. Neto, A.M.F, Galerne, Y, Levelut, A.M, Liebert, L, Pseudolamellar ordering in uniaxial and biaxial lyotropic nematics: A synchrotron X-ray diffraction experiment, Journal de Physique Lettres, 1985, 46 (11), 409-506.

3. Galerne, Y, Neto, A.M.F, Liébert, L, Microscopical structure of the uniaxial and biaxial lyotropic nematics, Journal of Chemical Physics, 1987, 87 (3), 1851-1856.

4. Akpinar, E, Reis, D, Yildirim, M, Neto, A.M.F, New lyotropic mixtures with non-chiral $\mathrm{N}$-acylamino acid surfactants presenting the biaxial nematic phase investigated by laser conoscopy, polarized optical microscopy and X-ray diffraction, Materials, 2014, 7 (6), 4132-4147.

5. Lavrentovich, O.D, Looking at the world through liquid crystal glasses, Contemporary Mathematics, 2012, 577, 25-45.

6. Lawson, K.D, Flautt, T.J, Magnetically oriented lyotropic liquid crystalline phases, Journal of American Chemical Society, 1967, 89, 5489-5491

7. Rosevear, F.B, The mesomorphic phases of surfactant compositions, Journal of the Society of Cosmetic Chemists, 1968, 19, 581-594.

8. Yu, L.J, Saupe, A, Observation of a biaxial nematic phase in potassium laurate-1-decanol-water mixtures, Physical Review Letters, 1980, 45, 1000-1003. 
9. Akpinar, E, Reis, D, Neto, A.M.F, Effect of Hofmeister anions on the existence of the biaxial nematic phase in lyotropic mixtures of dodecyltrimethylammonium bromide/sodium salt/1 dodecanol/water, Liquid Crystals, 2015, 42 (7), 973-981.

10. Galerne, Y, Marcerou, J.P, Temperature behavior of the orderparameter invariants in the uniaxial and biaxial nematic phases of a lyotropic liquid crystal, Physical Review Letters, 1983, 51, 2109 2111.

11. Freiser, M.J, Ordered states of a nematic liquid. Physical Review Letters, 1970, 24, 1041-1043.

12. Alben, $\mathrm{R}$, Phase transitions in a fluid of biaxial particles. Physical Review Letters, 1973, 30, 778-781.

13. Shih, C.S, Alben, R, Lattice model for biaxial liquid crystals, Journal of Chemical Physics, 1972, 57, 3055-3061.

14. de Gennes, P.G, Prost, J, The physics of liquid crystals, Clarendon Press: Oxford, UK, 1993. Chapter 1.

15. Neto, A.M.F, Salinas, S.R.A, The physics of lyotropic liquid crystals: phase transitions and structural properties, Oxford University Press: Oxford, UK, 2005, p 24.
16. Akpinar, E, Otluoglu, K, Turkmen, M, Canioz, C, Reis, D, Neto, A.M.F, Effect of the presence of strong and weak electrolytes on the existence of uniaxial and biaxial nematic phases in lyotropic mixtures, Liquid Crystals, 2016, 43 (11), 1693-1708.

17. Berejnov, V.V, Cabuil, V, Perzynski, R, Raikher, Y.L, Lysenko, S.N, Sdobnov, V.N, Lyotropic nematogenic system potassium laurate-1-decanol-water: method of synthesis and study of phase diagrams, Crystallography Reports, 2000, 45(3), 493-500.

18. Akpinar, E, Reis, D, Neto, A.M.F, Lyotropic mixture made of potassium laurate/1-undecanol/ $\mathrm{K}_{2} \mathrm{SO}_{4}$ /water presenting high birefringences and large biaxial nematic phase domain: a laser conoscopy study, European Physical Journal E, 2012, 35, 50 (19).

19. Akpinar, E, Canioz, C, Turkmen, M, Reis, D, Neto, A.M.F, Effect of the surfactant alkyl chain length on the stabilisation of lyotropic nematic phases, Liquid Crystals, 2018, 45 (2), 219-229. 